\title{
Current Trend in fabricating a Full Ceramic Restoration for Discolored Anterior Teeth: A Dental Laboratory Survey
}

\author{
${ }^{1}$ Naisargi P Shah, ${ }^{2}$ Aditi JP Jain, ${ }^{3}$ Sabita M Ram, ${ }^{4}$ Jyoti B Nadgere
}

\begin{abstract}
Aim: The aim of the survey conducted in dental laboratories was to evaluate the current trend in fabricating a full ceramic restoration for discolored anterior teeth through a questionnaire and personal interaction with the dental technician.
\end{abstract}

Materials and methods: The survey questionnaire consisted of 20 questions aimed toward the current trend in fabricating a full ceramic restoration for discolored anterior teeth. This questionnaire was validated by a panel of senior prosthodontists and technicians and was sent to 32 dental technicians in Mumbai and Navi Mumbai.

Results and conclusion: The results of the study showed that majority of the dental technicians preferred taking the stump shade themselves by visiting the clinic or inviting the patient to the laboratory. Majority of the dental technicians preferred using lithium disilicate material and its medium opacity ingots to fabricate restorations by the layering technique for masking discolored anterior teeth. Majority of the technicians believed that their dentists did not use try-in paste prior to luting of the restoration.

Clinical significance: This survey would help to improve the rapport between the clinicians and dental laboratories and optimize the esthetics of the restorations fabricated for anterior discolored teeth.

Keywords: Communication, Discolored teeth, Ingots, Lithium disilicate, (IPS e.max), Stump shade, Try-in paste.

How to cite this article: Shah NP, Jain AJP, Ram SM, Nadgere JB. Current Trend in fabricating a Full Ceramic Restoration for Discolored Anterior Teeth: A Dental Laboratory Survey. J Contemp Dent 2016;6(3):161-165.

Source of support: Nil

Conflict of interest: None

\section{INTRODUCTION}

One of the areas not often spoken about is the communication between the dentist and the dental technician.

\footnotetext{
${ }^{1,3,4}$ Professor, ${ }^{2}$ Postgraduate Student

${ }^{1-4}$ Department of Prosthodontics, Crown and Bridge, Mahatma Gandhi Mission's Dental College and Hospital, Navi Mumbai Maharashtra, India

Corresponding Author: Naisargi P Shah, Professor, Department of Prosthodontics, Crown and Bridge, Mahatma Gandhi Mission's Dental College and Hospital, Navi Mumbai, Maharashtra, India e-mail: drnaisargi69@gmail.com
}

This has become very important in imparting high-level esthetics to the patient. ${ }^{1}$ The dentist is to the dental technician what the architect is to the builder. In order to obtain the optimum esthetic results, it is essential that the dentist and dental technician work together effectively as a team. Each has a major role in indirect restorative dentistry, which is to replicate natural function, esthetics, and transform that into a sound restoration. The combination of the clinical experience, skill, and treatment planning by the dentist along with the technical expertise, knowledge of the properties of materials, and the ability to translate two-dimensional (2D) design diagrams and written instructions into the three-dimensional (3D) reality helps produce the best esthetic results. ${ }^{2}$

Restoring discolored anterior teeth poses a serious problem in achieving good esthetics when restored with all-ceramic crowns. ${ }^{3}$ Selecting the material of the restoration, shade, and communicating it to the dental technician presents an even bigger challenge. ${ }^{4}$ It is often difficult to communicate the various nuances of color to the technician with a single work order. The color replication process for dental porcelain comprises the shade selection phase followed by shade duplication. ${ }^{5}$ Shade selection is done by visual methods or by using modern technologies like colorimeters, spectrophotometers, and digital imaging systems..$^{6-8}$ This information is then conveyed to the dental technician in a manner that would appropriately help them to replicate even the smallest details in order to fabricate an esthetically sound restoration. The communication between the clinician and technician entails a thorough transfer of information, including the shade selected, the stump shade, material to be used for fabrication, functional components, occlusal parameters, phonetics, and esthetics.

This survey was, therefore, conducted to gather information from dental technicians in order to understand their outlook on the choice of materials they use and the method of fabrication of restorations for discolored anterior teeth. This survey would throw light on the step-bystep procedure commencing from the initial consultation, through treatment planning, and provisionalization to final placement. This, in turn, would improve the interaction between the clinicians and dental technicians and 
optimize and enhance the esthetics of the restorations fabricated for anterior discolored teeth.

\section{MATERIALS AND METHODS}

A survey was conducted among the dental technicians of Mumbai and Navi Mumbai fabricating all-ceramic restorations. This survey was approved by the Research Committee and Institutional Ethics Committee. The survey questionnaire consisted of 20 questions aimed toward the current trend in fabricating a full ceramic restoration for discolored anterior teeth. This questionnaire was validated by a panel of experts and was sent to 32 dental technicians in Mumbai. The dental laboratories chosen were both small, personalized labs and industrial labs with multiple technicians. The dental labs with more than 5 years of experience in carrying out all-ceramic work were chosen. The forms were received and a telephonic dialogue with the dental technicians was carried out to understand their perspective of fabrication of ceramic crowns, and then the results were analyzed.

\section{SURVEY FORM}

\section{The Current Trend in fabricating a Full Ceramic Restoration for Discolored Anterior Tooth: Dental Laboratory Survey}

1. Location of the laboratory

- urban

- rural

2. How old is the laboratory?

- $>5$ years

- $>10$ years

3. Since how many years have you been fabricating allceramic restorations?

- <5years

- >5years

4. How many dentists does your laboratory provide service to?

- Less than 20

- More than 20

5. Are you dictated by your dentist's opinion in selection of all-ceramic material for anterior restorations?

- Yes

- No

6. Would you like to select the shade yourself by calling the patient to the operatory or going to the dental clinic?

- Yes

- No

7. If yes, at what stage would you like the patient's presence in the laboratory?

- At the time of ingot selection

- At the time of layering
8. Does the dentist provide you with the stump shade for discolored tooth?

- Yes

- No

- Not always

9. If yes, how does your dentist select the stump shade?

- Shade guides

- Digital methods

- Camera imaging system

10. How does the dentist communicate the stump shade with the laboratory?

- Oral

- Written

- Photographs sent electronically (E-mail/ WhatsApp)

11. Which method of communication do you prefer?

- Oral

- Written

- Photographs sent electronically (E-mail/ WhatsApp)

12. Do you utilize this communication for selecting the shade of the ingot?

- Yes

- No

13. Which material do you prefer for anterior restorations for discolored teeth?

- IPS e.max

- Zirconia

- IPS Empress

14. If IPS e.max, which of the following ingot would you suggest for anterior discolored teeth?

- High opacity

- Medium opacity

- Low translucency

- High translucency

15. Based on the feedback of the dentist, which of the following ingot will give better esthetics?

- High opacity

- Medium opacity

- Low translucency

- High translucency

16. If using e.max, which technique do you use for fabricating anterior restorations from medium opacity and high opacity?

- Layering technique

- Cut-back technique

17. If using e.max, which technique do you use for fabricating anterior restorations from low translucency and high translucency?

- Layering technique

- Cut-back technique 
18. Would you take into consideration the shade of the cement which the dentist will be using for luting the restoration?

- Yes

- No

19. Does your dentist use try-in pastes for the selection of the final cement?

- Yes

- No

20. Do you fabricate the die of the stump shade before finalizing the shade of the restoration?

- Yes

- No

\section{RESULTS}

Questionnaires were sent to 32 dental laboratories; only 29 responded. Data were fed to the Statistical Package for the Social Sciences (SPSS) software version 6.0 and statistical analysis was done. Frequencies and percentages of all the questions and answers were calculated. The results of this study showed that $74.2 \%$ of the dental technicians preferred that their dentists send the patient to the laboratory to visualize the shade or approach them to visit the clinic on the day of shade selection (Graph 1). About $55.2 \%$ of the dental technicians preferred taking the shade at the time of layering and $44.8 \%$ at the time of ingot selection (Graph 1). Only 33.3\% dentists provided the stump shade to the dental technician (Graph 1). Out of the dentists who provided the stump shade, $80 \%$ of them used the VITA Shade guide, $10 \%$ used the digital devices, and another $10 \%$ used the camera imaging system to convey the stump shade to the dental technician (Graph 2).

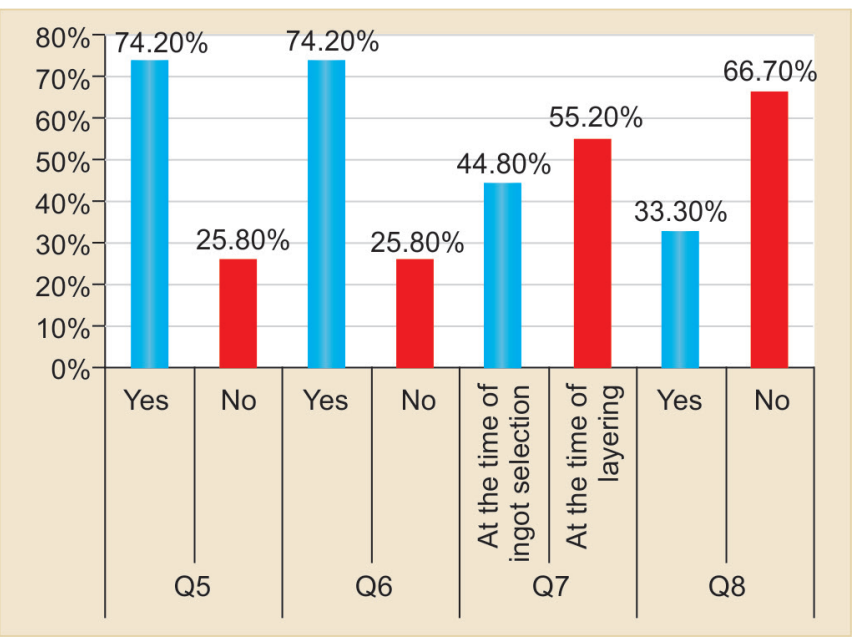

Graph 1: Q5 TO Q8

About $50 \%$ dentists communicated the stump shade to the laboratory through a written prescription, and $21.43 \%$ communicated the shade using photographs (Graph 2).

About $21.43 \%$ dental technicians preferred both the written prescription and photographs sent electronically for better understanding (Graph 2). About 50\% dental technicians preferred using lithium disilicate material. About $42.31 \%$ dental technicians preferred using zirconia for discolored teeth (Graph 3). Of those dental technicians who preferred using lithium disilicate material (IPS emax), 55.17\% used the medium-opacity ingot to fabricate the restorations for masking discolored anterior teeth (Graph 3). Based on the feedback from the dentists by the technicians, $53.57 \%$ dental technicians believed that medium opacity gave better esthetics than the highopacity, low-translucency, and high-translucency ingots (Graph 3). About $82.76 \%$ dental technicians used the layering technique for fabricating anterior restorations

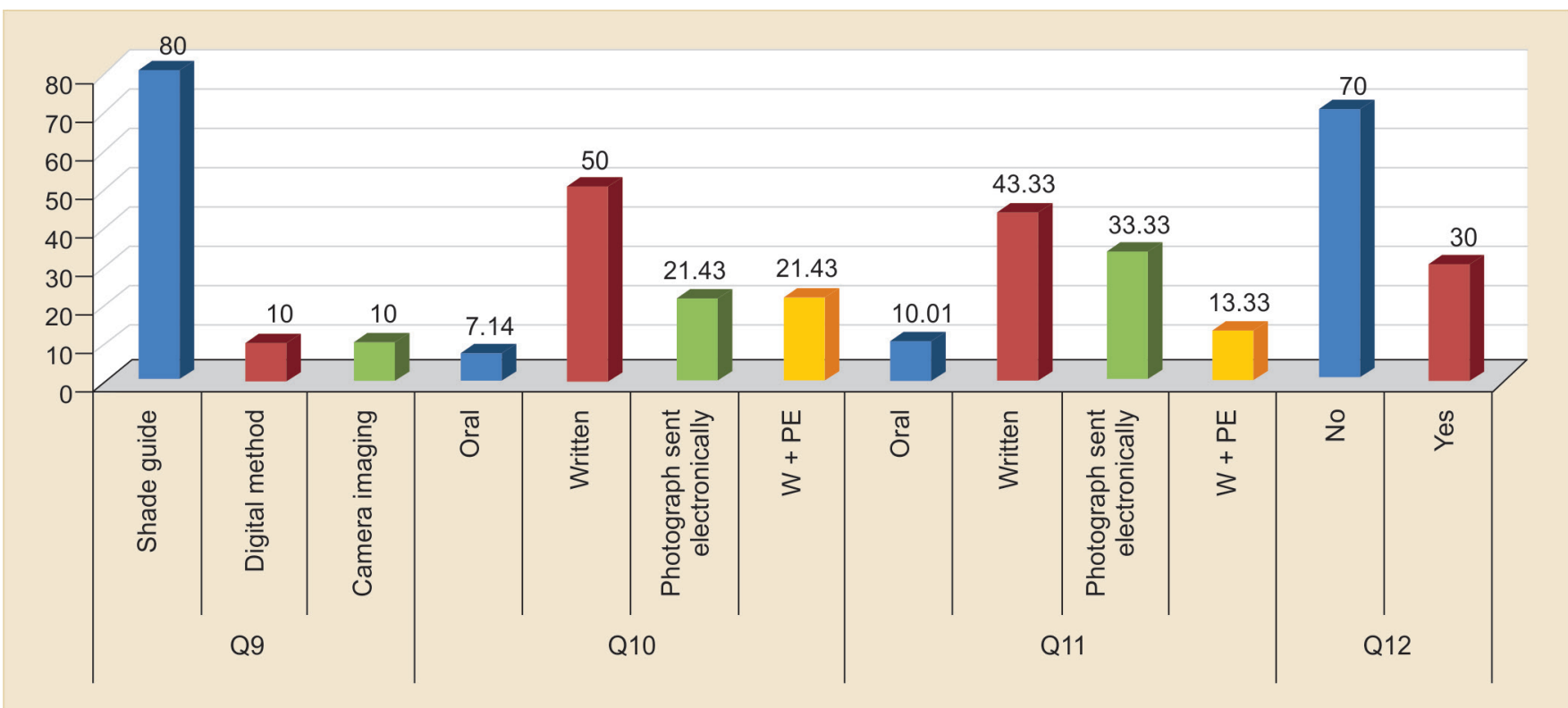

Graph 2: Q9 TO Q12 


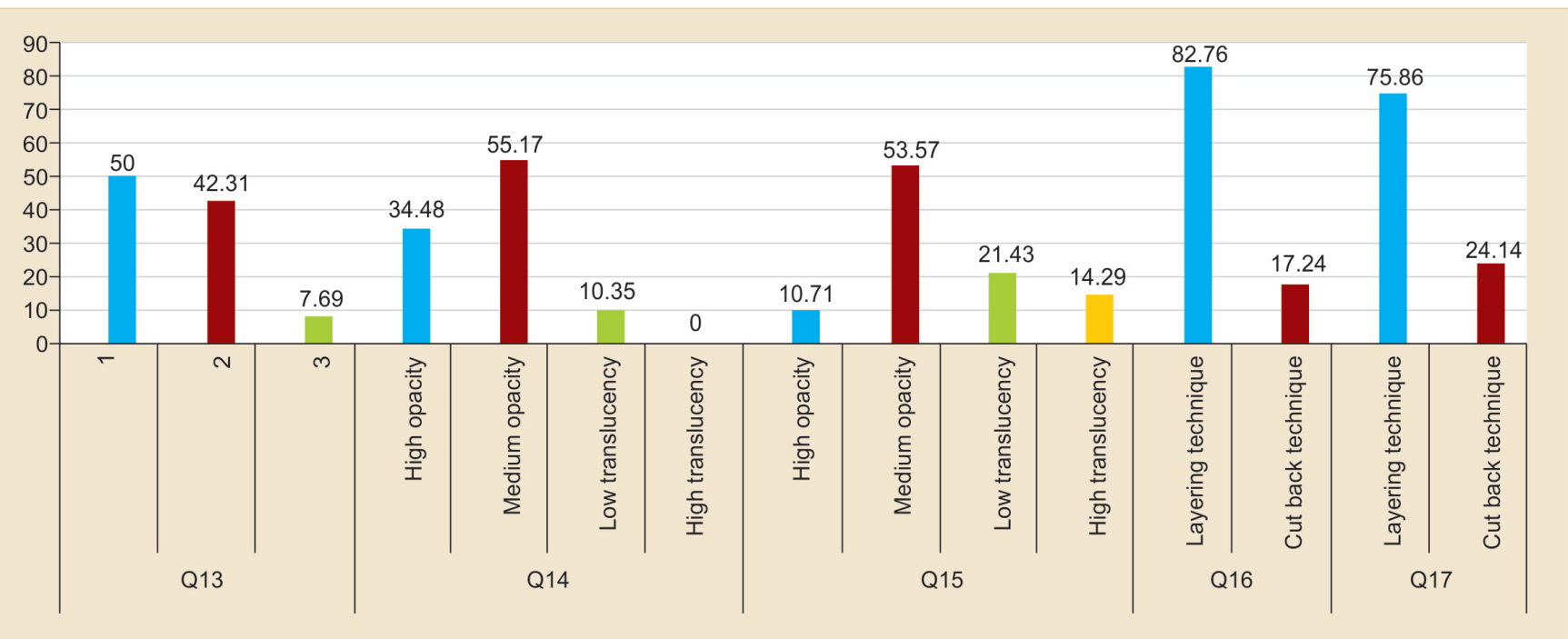

Graph 3: Q13 TO Q17

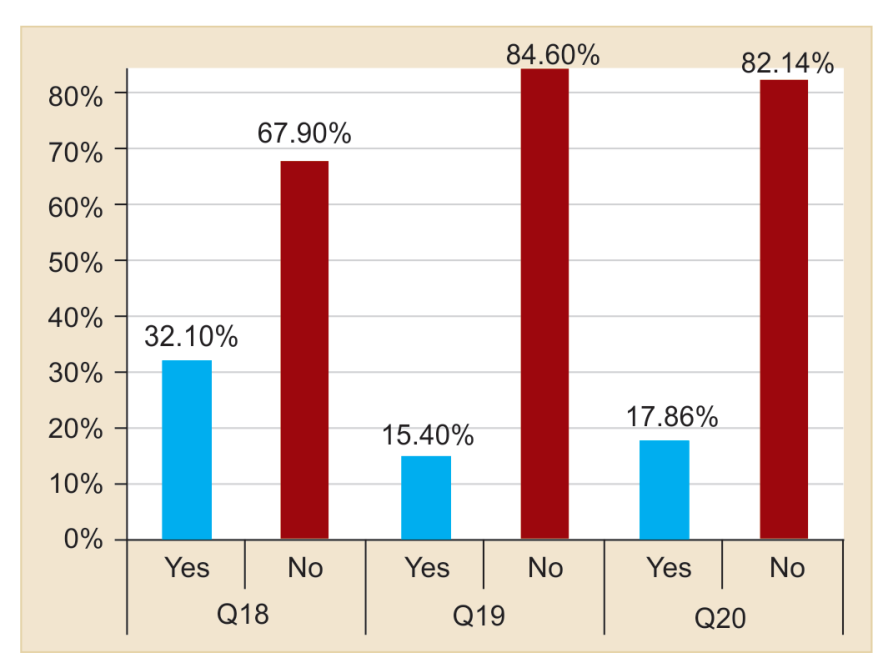

Graph 4: Q18 TO Q20

using medium- and high-opacity ingots (Graph 3). About $75.86 \%$ dental technicians used the layering technique for fabricating anterior restorations using low- and hightranslucency ingots (Graph 3).

About 32.1\% dental technicians took the shade of the cement into consideration while fabricating the restoration (Graph 4). About $84.60 \%$ dental technicians stated that their dentists did not use the try-in paste before cementation (Graph 4). About 82.14\% dental technicians did not fabricate the die of the stump shade before finalizing the shade of the restoration (Graph 4).

\section{DISCUSSION}

After the survey was filled, it was discussed with the laboratory technicians to understand their point of view for every question. This survey was carried out in various laboratories across Mumbai and Navi Mumbai. Majority of these laboratories were more than 10 years old; hence, they were highly experienced and precise in their work.
They have been fabricating all-ceramic restorations for more than 5 years, and they catered to more than 20 dentists. About $74.2 \%$ dental technicians were dictated by the dentists' opinion while fabrication of the restorations, and the rest of the technicians preferred taking the decision on their own or putting in their own inputs in order to enhance the esthetic results. Hatzikyriakos et $\mathrm{al}^{9}$ had carried out a survey in which $30 \%$ of shade selection was undertaken by the dental technicians. In the present survey, $74.2 \%$ dental technicians stated that they preferred taking the stump shade by themselves, though it was not always possible due to the unwillingness of the patient to spend time going to the lab, or the location of the lab not being in close proximity.

About 55.2\% dental technicians took the stump shade at the time of layering of the ingot in order to replicate even the smallest details and create a life-like appearance of the restoration. The stump shade should have been taken prior to layering in order to select the appropriate shade and translucency of the ingot. The stump shade is an extremely vital information which should be conveyed to the dental technician, especially where discolored teeth have to be restored and the discoloration has to be masked; but, in this survey, it was found that the majority of the dentists did not provide the stump shade to the dental technicians. Some of the dentists who provided this information used the VITA shade guide to convey the stump shade, but it is not an ideal shade guide for determining the shade of discolored teeth. There are special shade guides available, which help in estimating the stump shade of discolored teeth. Shade guides are not a perfect representation of what is actually seen, but are close enough to identify a range of tooth colors. Eyes are still the best tool for identifying and communicating the correct dental shade. It is 
always best to get the patient to the dental laboratory and have the shade taken, particularly for those cases where achieving esthetics is critical.

Berry et $\mathrm{al}^{10}$ had carried out a study in which the laboratory prescription and the telephone were the main communication tools used between the dentists and the dental technicians. The results of this study showed that $50 \%$ dentists communicated through a written prescription with the dental technician. Many clinicians send their cases without a picture, so there are limitations when trying to create the best shade match for the restorations. ${ }^{11}$ In the present survey, $43.33 \%$ dental technicians preferred using the written prescription and $33.33 \%$ preferred the use of shade guides in conjunction with digital photography. ${ }^{12}$

In the current survey, 50\% dental technicians preferred using lithium disilicate material IPS emax for masking discoloration. Though there are many allceramic material choices available, favorable physical and esthetic properties make glass-based lithium disilicate an excellent material for anterior restorations. This material currently has more options available for shade as well as light transmittance than any other available ceramic system. ${ }^{13,14}$ There are currently 47 ingot selections available for the e.max Press system (Ivoclar Vivadent). The selection of an appropriate ingot will allow for the masking of a discolored tooth and can achieve optical uniformity between multiple teeth of varying colorations. In this study, majority of the dental technicians used the medium-opacity ingot. The reasonable explanation to this could be that high-opacity ingot would make the restoration look highly opaque in the anterior esthetic zone and the low-translucency and high-translucency ingot would be unable to conceal the discoloration. So, the mediumopacity ingot would create a visual harmony between the natural tooth and the restoration on the discolored tooth.

Chadwick et $\mathrm{al}^{15}$ stated that try-in pastes available match the shades of the luting agents that are used to finally cement the restoration in place. It is essential to gain the approval of the patient before final cementation takes place. Such restorations should, therefore, be tried by using a try-in paste. This allows both the dentist and the patient to evaluate the shape, chroma, hue, value, and translucency of the restorations to ensure that all esthetic expectations can be matched. Based on the feedback from dental technicians, only $15.4 \%$ of their dentists used the try-in paste for selection of the final cement. Baldermos et $\mathrm{al}^{16}$ had carried out a study stating that there were significant differences in color between resin cements and their corresponding try-in pastes.

\section{CONCLUSION}

Through analysis of the observations in this survey and within the limitations of the study, the following conclusions can be drawn:
- Majority of the dental technicians preferred taking the shade themselves because the dentists do not communicate the stump shade correctly.

- Majority of the dental technicians preferred using lithium disilicate material IPS emax and its mediumopacity ingots to fabricate restorations by layering technique for masking discolored anterior teeth.

- Majority of the dental technicians believed that their dentists did not use try-in paste prior to luting of the restoration.

\section{REFERENCES}

1. Mhaske PN, Ram SM, Shah N, Nadgere JB. Minimally invasive approach to restore anterior spaces with porcelain laminate veneers. J Contemp Dent 2013 Jun; 3(2):108-111.

2. Davenport JC, Basker RM, Heath JR, Ralph JP, Glantz PO, Hammond P. Communication between the dentist and the dental technician. Br Dent J 2000 Nov;189(9):471-474.

3. Shah N, Nadgere J, Khanna T. Restoring maxillary lateral incisors with ceramic laminate veneers: an esthetic challenge. Int J Prosthet Dent 2010 Dec;1(1):15-16.

4. Edelhoff D, Brix O. Restoring severely discoloured anterior teeth using minimally invasive procedures. Int Dent 2011;1(3): $42-48$.

5. Wee AG, Monaghan P, Johnston WM. Variation in color between intended matched shade and fabricated shade of dental porcelain. J Prosthet Dent 2002 Jun;87(6):657-666.

6. Chu SJ, Trushkowsky RD, Paravina RD. Dental color matching instruments and systems. Review of clinical and research aspects. J Dent 2010;38 (Suppl 2):e2-e16.

7. Douglas RD, Steinhauer TJ, Wee AG. Intraoral determination of the tolerance of dentists for perceptibility and acceptability of shade mismatch. J Prosthet Dent 2007 Apr;97(4):200-208.

8. Chen H, Huang J, Dong X, Qian J, He J, Qu X, Lu E. A systematic review of visual and instrumental measurements for tooth shade matching. Quintessence Int 2012 Sep;43(8):649-659.

9. Hatzikyriakos A, Petridis HP, Tsiggos N, Sakelariou S. Considerations for services from dental technicians in fabrication of fixed prostheses: a survey of commercial dental laboratories in Thessaloniki, Greece. J Prosthet Dent 2006 Nov;96(5):362-366.

10. Berry J, Nesbit M, Saberi S, Petridis H. Communication methods and production techniques in fixed prosthesis fabrication: a UK based survey. Part 1: communication methods. Br Dent J 2014 Sep;217(6):E12.

11. Kahng LS. Hidden effects of stump shades. Bench Dent 2010:2-3.

12. Mahn E. Dental photography. Part II protocol for shade taking and communication with the lab. Int Dent Aust;8(2):36-44.

13. Volker AR, Springs PA, Romanzi S. Treating discolored anterior teeth: ingot selection and cementation protocol for lithium disilicate restorations. Dent Today 2014;33(10):102.

14. Della Bona A, Kelly JR. The clinical success of all-ceramic restorations. J Am Dent Assoc 2008 Sep;139 (Suppl):8S-13S.

15. Chadwick RG, McCabe JF, Carrick TE. Rheological properties of veneer trial pastes relevant to clinical success. Br Dent J 2008 Mar;204(6):E11.

16. Baldermos LP, Keefe KL, Powers JM. Colour accuracy of resin cements and try-in pastes. Int J Prosthodont 1997 MarApr;10(2):111-115. 